\title{
Optical Properties of Rutile Single Crystals Grown Under Pressure by Tilting-Mirror-Type Floating Zone Method
}

\author{
M. A. R. Sarker ${ }^{\mathrm{a}, \mathrm{b}^{*}}$ \\ ${ }^{a}$ Center for Crystal Science and Technology, University of Yamanashi, 7-32 Miyamae, Kofu, \\ Yamanashi 400-8511, Japan \\ ${ }^{\mathrm{b}}$ Department of Physics, University of Rajshahi, Rajshahi-6205, Bangladesh
}

Received 21 May 2014, accepted in final revised form 29 March 2015

\begin{abstract}
Rutile single crystals with high quality and high transparency were grown successfully by tilting-mirror-type floating zone (TMFZ) technique. The grown crystals were characterized by polarized optical microscopy, X-ray diffractometer, UV-visible spectrophotometer and etch pits density measurement. The effect of applied pressure during growth on the optical properties and defect density of the rutile crystals were investigated. It was found that the rutile crystals grown under the high oxygen pressure are bright and transparent due to the lower concentration of defects, but rutile crystals grown at ambient or lower oxygen pressure exhibit deep blue color due to presence of defects such as dislocations and grain boundaries. The crystals grown under high oxygen pressure contains no low-angle grain boundaries, indicating that it can be used for high efficiency optical devices.
\end{abstract}

Keywords: Defects; Interfaces; Floating zone technique; Titanium compounds; Optical materials.

(C) 2015 JSR Publications. ISSN: 2070-0237 (Print); 2070-0245 (Online). All rights reserved. doi: http://dx.doi.org/10.3329/jsr.v7i1-2.18927 J. Sci. Res. 7 (1-2), 1-9 (2015)

\section{Introduction}

Rutile $\left(\mathrm{TiO}_{2}\right)$ is a versatile material with technological applications as photocatalist, photovoltaic material, optical coating and structural ceramic. It has excellent optical, mechanical and chemical properties, and the demand for rutile single crystals has therefore been increasing to use as polarizer in a variety of optical devices because of their large refractive indices and birefringence [1]. It is of urgent necessity to grow rutile single crystals of high optical quality. The floating zone (FZ) method is one of the most promising techniques, which replace the Vemeuil method, to grow rutile single crystals. Rutile single crystals were conventionally manufactured by the Verneuil method for gemstones [2]. However, the quality of Verneuil-grown crystals is usually poor, since the crystals undergo quenching, which induces stress birefringence [3]. At present,

\footnotetext{
* Corresponding author: razzaquephy@ru.ac.bd
} 
commercially available rutile single crystals grown by the Verneuil method, are generally not good enough to use in optical devices because of their dislocation density due to presence of Magnel phases $\left(\mathrm{TiO}_{2-\delta}\right)$ i.e. oxygen deficient phases. The optical quality of the FZ-grown crystals was superior to that of commercially available Verneuil-grown rutile crystals $[4,5]$. Therefore, demands for the optical communication devices have stimulated the application of a floating zone method for the growth of rutile single crystals although conventional-FZ grown rutile crystals are not completely free of defects [5].

Floating zone (FZ) method is a powerful technique for growth of single crystals without contaminations because it is a crucible free zone melting method whereas in the conventional FZ method some defects occur for shape of the molten zone. The shapes of both the melt-feed and the melt-crystal interfaces are convex in the FZ method. The defects are generally caused by the low thermal conductivity on the convex solid-liquid (crystal-melt) interface during the growth of rutile crystals [6]. It has been reported that interface shape affects the density of defects in crystals such as etch pits [7]. An extremely convex interface creates thermal stress that causes defects [8]. Kinoshita et. al. showed that the low-angle grain boundaries are markedly reduced when the solid-liquid interface is almost flat during crystal growth [9]. A slightly convex interface towards melt is better for improving the quality of crystals [10]. Recently, we reported that the interface shape can be controlled by tilting the mirror in the tilting-mirror-type floating zone (TMFZ) method as shown in Fig.1 [11]. It was revealed that the quality of the TMFZ-grown crystals increases with increase of tilting angle [12] and growth diameter [13]. However, the defects due to oxygen deficiency still occur in the TMFZ grown crystals.

In this study, we try to grow high quality rutile single crystal by TMFZ method for optical device applications. The TMFZ method is an outstanding way of growing highly pure single crystals especially for oxides. The rutile single crystals were grown under pressure by this method. It was considered that the effects of high oxygen pressure on the growth of single crystals could deteriorate the oxygen coming out of the molten zone and compensate for the vacancy sites physically. Therefore, the high quality rutile single crystals would be obtained.

\section{Experimental}

A new tilting-mirror-type image furnace (Crystal Systems Corporation; model TLFZ4000-H -VPO) was developed for our growth experiment. The tilting angle $(\theta)$, as shown in Fig. 1, can be changed to up to $30^{\circ}$ by a motor drive control. High-purity rutile powder ( $>99.99 \%$ ) was put into rubber tubes to form a rod shape and pressed at a pressure of up to $3.0 \times 10^{8}$ Pa using a cold isostatic pressing machine (Nikkiso Co., Ltd.: model CL3-2260). The rods were sintered at $1600^{\circ} \mathrm{C}$ for $12 \mathrm{~h}$ in an oxygen flow. The sintered rods were zone-passed in a $\mathrm{CO}_{2}$ flow at a growth rate of $25 \mathrm{~mm} / \mathrm{h}$ using a standard type four mirrortype-image furnace (Crystal Systems Corporation: model FZ-4000H). The zone-passed feed rods were typically $10-12 \mathrm{~mm}$ in diameter and $80-100 \mathrm{~mm}$ in length. 
The applied conditions for every growth experiment were a growth rate of $5 \mathrm{~mm} / \mathrm{h}$, a feeding rate of $5 \mathrm{~mm} / \mathrm{h}$, upper shaft rotation of $3 \mathrm{rpm}$, lower shaft rotation of $50 \mathrm{rpm}$, a growth direction of $<001>$ using a rutile seed crystal. The crystals were grown with oxygen pressures of $1,3,5$, and $8 \mathrm{~atm}$. The tilting angle, $\theta$ was $20^{\circ}$. It was reported that this tilting condition was best for growth of good quality large diameter single crystals with most stabilized molten zone. The grown crystal was checked as single crystal by Laue technique using Rigaku X-ray diffractmeter. The grown crystals were cut perpendicular to the growth direction in the range $23-25 \mathrm{~mm}$ from the seeding point. Then the crystals were cut parallel to (110) and the surface was polished like mirror. The polished surfaces were observed by optical microscope (Olympus U-MSSPG Japan) to investigate the phase purity and optical quality. The polished samples were soaked in a mixture of (NH4) ${ }_{2} \mathrm{SO}_{4}$ and $\mathrm{H}_{2} \mathrm{SO}_{4}$ solutions (1:1 weight ratio) for $3 \mathrm{~h}$ at $300^{\circ} \mathrm{C}$ to etch their surfaces. The polished samples of $\sim 1.5 \mathrm{~mm}$ in thickness were characterized by UVVisible spectrometer (JASCO: V-660).

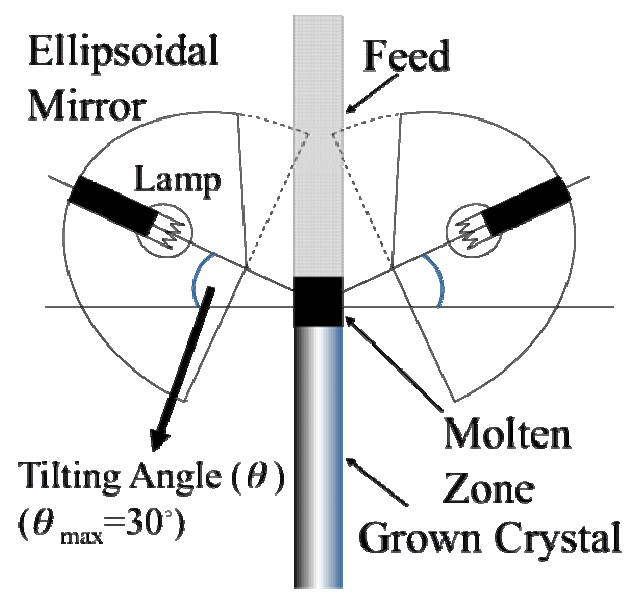

Fig. 1. Schematic illustration of the tilting mirror type image furnace (The definition of tilting angle $(\theta)$ is given).

\section{Results and Discussions}

\subsection{Bulk properties of rutile}

After flushing inside a quartz tube with flowing oxygen, oxygen pressures of 1, 3, 5 and 8 atm were applied and then the crystal was grown. The as-grown single crystals were very different from each other when they were observed by the naked eye for coloring indication as shown in Fig. 2 (a-e). The rutile crystal grown in an atmospheric pressure by conventional FZ method is dark blue while for TMFZ method it is light blue. However, the rutile crystals grown under high oxygen pressure by TMFZ method exhibit a transparent amber color as shown in Fig. 2 (c-e). Each grown crystals were equally dark 
or bright from shoulder to tail part. The observations describe the color of crystals as a function of oxygen pressure applied on the molten zone during growth. The annealed rutile crystal being more transparent reduced the amount of oxygen vacancy, and delocalized its original color [14]. However, the annealed sample is not good for optical applications because of the low angle grain boundary resulting from polygonalized dislocations [6]. The Laue photograph of the grown crystal was taken to confirm as single crystal as shown in Fig. 3. The X-ray diffraction Laue analysis of the grown crystals confirmed that they were single crystals and their growth directions were the [001] direction perpendicular to the c-axis. This analysis also stated that the grown crystals structure was tetragonal.

(a)

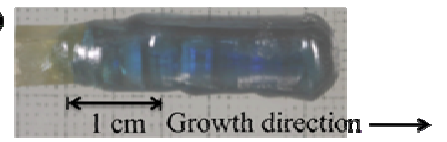

(b)

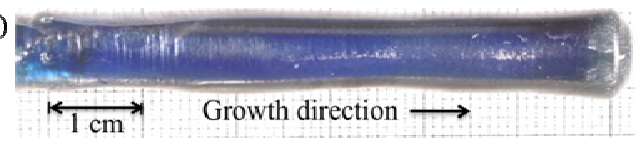

(c)
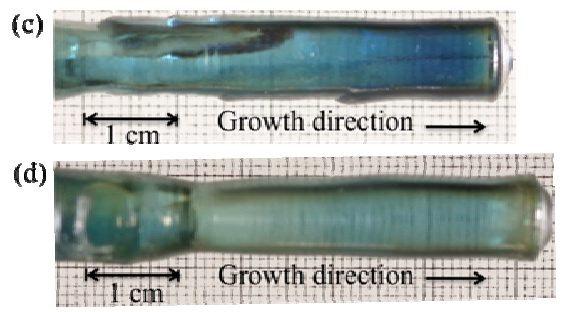

(c)

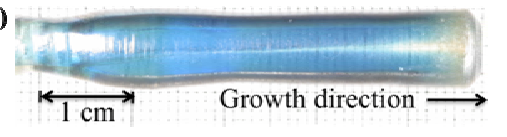

Fig. 2. Photographs of the rutile crystals grown at (a) $1 \mathrm{~atm}$ by conventional FZ (b) $1 \mathrm{~atm}$ (c) $3 \mathrm{~atm}$ (d) $5 \mathrm{~atm}$ (e) $8 \mathrm{~atm}$ by TMFZ method

Fig. 3. X-ray diffraction Laue photograph of the rutile single crystals.

The grown crystals sliced at $22-25 \mathrm{~mm}$ from the seeding point along perpendicular to the growth direction. The wafers were mechanically polished like mirror using diamond 
paste. The polished wafers were investigated by polarized optical microscope. Fig. 4 (a-e) shows micrograph of the cross-section of crystals grown by conventional FZ at an atmospheric pressure and by TMFZ at 1, 3, 5 and 8 atmospheric pressure. The conventional FZ crystals contain some low-angle grain boundaries. The TMFZ crystals grown at 1 atm contains a little low-angle boundaries but some segregated phases observed in this crystal as shown in Fig. 4(b). Fig. 4 (c-e) reveal that the crystals grown by TMFZ method at $3-8$ atm pressure did not contain any low-angle grain boundaries where low-angle grain boundaries are known as the critical defects in optical applications. The mirror-tilting effects in the TMFZ method as well as pressure effect during growth improve the quality of the rutile crystals for high efficiency optical applications.

(a)

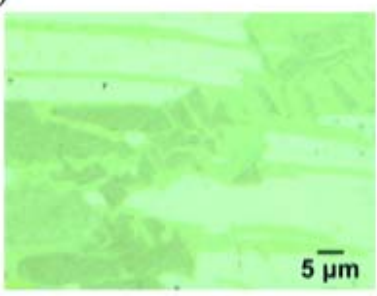

(c)

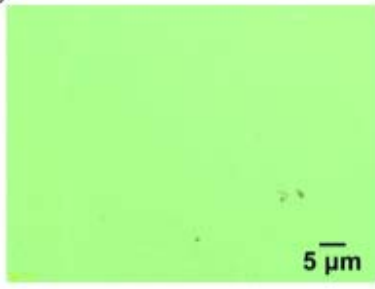

(b)

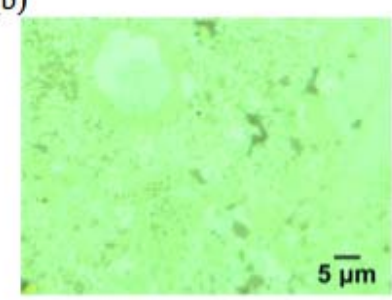

(d)

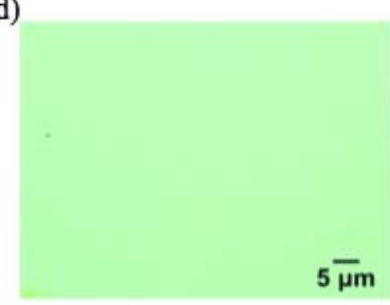

(e)

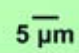

Fig. 4. (a) Optical micrographs of polished cross-section of the rutile crystals grown at (a) 1 atm by conventional FZ (b) 1 atm (c) 3 atm (d) 5 atm (e) 8 atm by TMFZ method.

\subsection{Defect analysis}

In the FZ method, the quality of the grown crystal markedly affected by the crystal-melt interface. Kitamura et al. found that line defects propagate to the edge of a crystal if the crystal-melt interface is more convex toward the melt [7]. Chiang et al. showed that showed that a slightly convex interface towards the melt is better for growth of high quality single crystals [10]. In our recent study, it was found that the crystal-melt interface 
shape during FZ growth can be controlled by tilting the mirror in TMFZ method to improve the crystal quality [12]. In this study, we further improve the quality of the crystals by applying the pressure on the molten zone during growth by TMFZ method. To investigate the inside of quality of grown crystals, we analysis the defects measuring etch pits density (EPD) because Hirthe et al. showed that the etch pit corresponds to dislocations [15] and Kinoshita et al. showed that etch pits arise in the presence of lowangle grain boundaries [9].

Fig. 5 (a-e) shows optical microphotographs of the samples on (110) surface after chemical etching. The observed etch pits are clearly more in the crystal grown by conventional FZ method compared with that in the crystal grown by TMFZ method as shown in Fig. 5 (b-e). In case of TMFZ crystals, the number of pits decreased with increasing oxygen pressure during growth. The etch pit densities (EPDs) determined in the rutile crystals grown by conventional FZ method at an atmospheric pressure are $1.393 \times 10^{4} \mathrm{~cm}^{-2}$ at the center and $4.7 \times 10^{4}$ at the periphery (4 mm from center). The estimated EPDs of the rutile crystals grown by the TMFZ $\left(\theta=20^{\circ}\right)$ method at an atmospheric pressure are $3.47 \times 10^{3} \mathrm{~cm}^{-2}$ at center and $9.25 \times 10^{3} \mathrm{~cm}^{-2}$ at periphery. So EPD was reduced by around 4 times at center and 5 times at periphery with TMFZ growth.
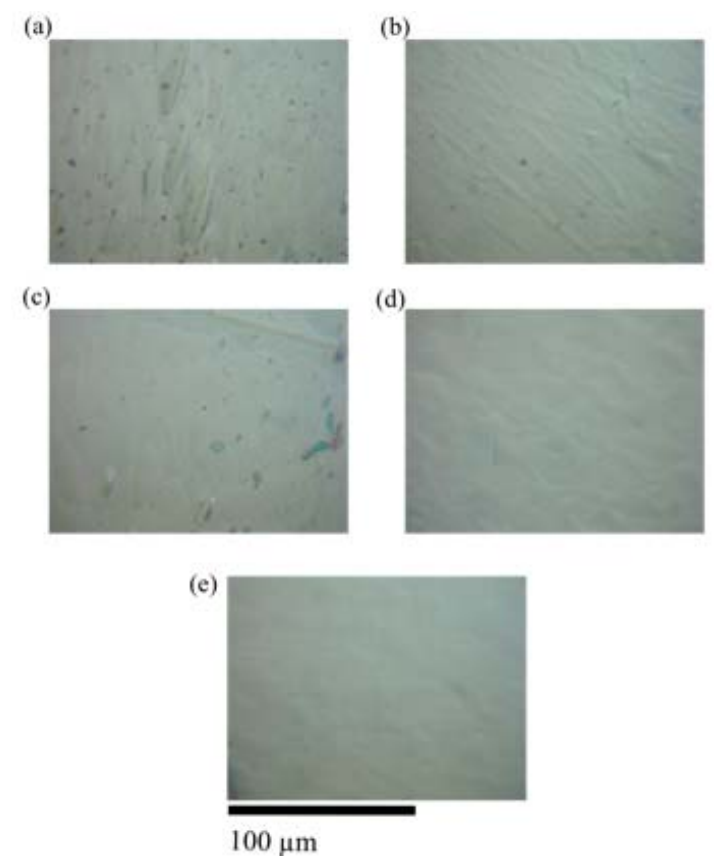

Fig. 5. Optical microphotographs of etch pits on (110) of rutile single crystals grown at (a) $1 \mathrm{~atm}$ by conventional FZ (b) 1 atm (c) 3 atm (d) 5 atm (e) 8 atm by TMFZ method.

The EPDs also reduced for pressure effects in the TMFZ growth. Fig. 6 (a-e) shows the EPDs on (110) as functions of the radius position $(X)$ of the crystals grown at various oxygen pressures by TMFZ method. For all oxygen pressures, the EPDs are higher at the 
periphery than at the center. The EPDs decreased significantly for applied pressure both at center and periphery of the crystal grown by TMFZ method. As the etch pits are associated with dislocations and grain boundaries, the quality of the rutile crystals can be improved by tilting the mirror in TMFZ method as well as by applying oxygen pressure during growth. These results reveal that TMFZ is a powerful method for growth of high quality single crystals for optical device applications.

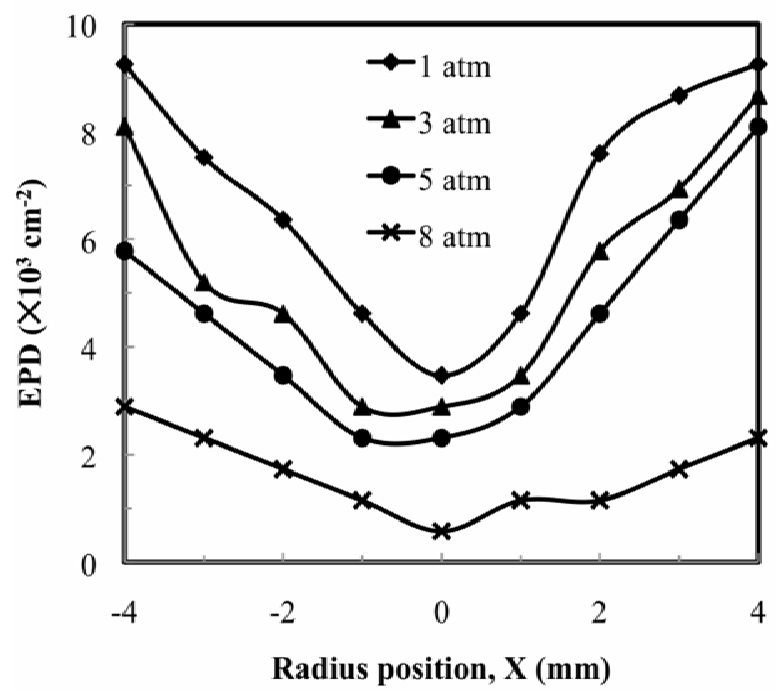

Fig. 6. Distributions of etch pit density as a function of radius position $(X)$ of crystals grown at different atmospheric pressure using TMFZ method.

\subsection{Optical properties of rutile}

Rutile single crystal is the most important material for optical device applications such as polarizer, isolator, refractor etc that requires the high quality crystals with high transmittance. Hence the transmittance was also measured as a further criterion for optical applications. The transmittance data of the disc like samples with $1.5 \mathrm{~mm}$ in thickness is shown in Fig. 7. The transmittance of the rutile crystals grown by conventional FZ method under $1 \mathrm{~atm}$ pressure is $18.5 \%$ while that in case of TMFZ is $32.5 \%$.

The transmittance increased for the pressure effect in the TMFZ grown rutile $\mathrm{TiO}_{2}$. Rutile crystals grown under high oxygen pressure exhibited more than $43 \%$ of the transmittance in the wavelength range longer than $400 \mathrm{~nm}$. This result is consistent with the color change of grown crystals as shown in Figs. 2. This change corresponds to the change of oxygen deficiencies. The high oxygen pressure on the molten zone during growth suppressed the oxygen vacancy. The band edge is $420 \mathrm{~nm}$ for all samples, which is independent of the pressure effect. The transmission peak of the rutile crystal grown by conventional FZ is at $460 \mathrm{~nm}$ while that of rutile crystal grown by TMFZ method under one atm is at $472 \mathrm{~nm}$. The transmission peak of rutile crystals grown under $3 \mathrm{~atm}$, 5atm 
and $8 \mathrm{~atm}$ are at $483 \mathrm{~nm}, 484 \mathrm{~nm}$ and $581 \mathrm{~nm}$ respectively. The transmission peak of the rutile crystals shifted significantly towards higher wavelength region for pressure effect during growth. There is a sharp transmission peak at $710 \mathrm{~nm}$ and a sharp absorption peak at $856 \mathrm{~nm}$ in the TMFZ grown rutile crystals grown under 8 atm pressure. These are the chrematistic peaks of the rutile crystals for optical device applications.

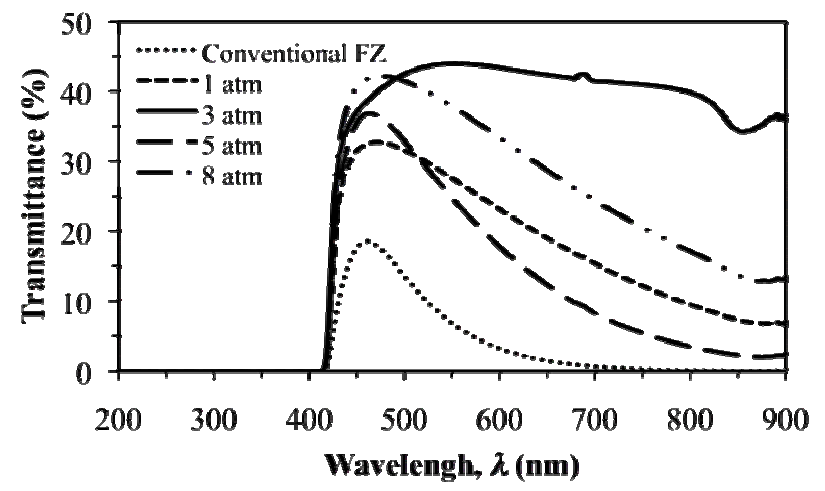

Fig. 7. Transmittance as a function of wavelength of light for the rutile crystals grown at (a) 1 atm by conventional FZ (b) 1 atm (c) 3 atm (d) 5 atm (e) 8 atm by TMFZ method.

\section{Conclusions}

High quality rutile single crystals, free from defects were successfully grown by infrared heating tilting-mirror-type floating zone (TMFZ) method. The rutile single crystals grown at $1 \mathrm{~atm}$ pressure by the conventional floating zone method are observed as dark blue colour where that crystals grown by the TMFZ method observed as light blue colour. The brightness of the grown crystals increases with increase of atmospheric pressure during growth by TMFZ method. It was observed that the colour was changed due to elimination of defects such as low-angle grain boundaries, oxygen vacancies and dislocation densities. The transmittance of the rutile crystals grown at 1 atm pressure by conventional FZ method is $18.5 \%$ whereas that of the TMFZ grown rutile crystal is $32.5 \%$. The transmittance of the TMFZ grown rutile crystal also found to be increases with increase of pressure during growth. The rutile single crystals of high transparency are the best for optical device applications.

\section{Acknowledgments}

This work was partially supported by the Nippon Sheet Glass Foundation for Materials Science and Engineering, the Sasagawa Postdoctoral Science Foundation Program (No. 13-001) of the Japan Science Society (JSS), Grant-in-Aid for Scientific Research (C) (No. 20550173) of Japan Society for the Promotion of Science (JSPS). 


\section{References}

1. M. Shirasaki, and K. Asama, Appl. Opt. 21, 4296 (1982). http://dx.doi.org/10.1364/AO.21.004296

2. Y. Nakazumi, Seramikkusu 3, 731 (1968).

3. Y. Nakazumi, K. Suzuki, and T. Yajima, J. Phys. Soc. Japan 17, 1806 (1962). http://dx.doi.org/10.1143/JPSJ.17.1806

4. M. Higuchi and K. Kodaira, J. Cryst. Growth 123, 495 (1992). http://dx.doi.org/10.1016/0022-0248(92)90611-L

5. J. K. Park, K. H. Kim, I. Tanaka, and K. B. Shim, J. Cryst. Growth 268, 103 (2004). http://dx.doi.org//10.1016/j.jcrysgro.2004.04.105

6. J. K. Park, K. B. Shim, K. W. Auh, and I. Tanaka, J. Cryst. Growth 237-239, 730 (2002). http://dx.doi.org//10.1016/S0022-0248(01)01989-3

7. K. Kitamura, S. Kimura, and S. Hosoya, J. Cryst. Growth 48, 469 (1980). http://dx.doi.org/10.1016/0022-0248(80)90044-5

8. M. Higuchi and K. Kodaira, Mater. Res. Bull. 29, 545 (1994). http://dx.doi.org/10.1016/0025-5408(94)90044-2

9. K. Kinoshita, and K. Sugii, J. Cryst. Growth 71, 283 (1985). http://dx.doi.org/10.1016/0022-0248(85)90082-X)

10. C. H. Chiang and J. C. Chen, J. Cryst. Growth 294, 323 (2006). http://dx.doi.org/10.1016/j.jcrysgro.2006.06.022

11. M. A. R. Sarker, S. Watauchi, M. Nagao, T. Watanabe, I. Shindo, and I. Tanaka, J. Cryst. Growth 312, 2008 (2010). http://dx.doi.org/10.1016/j.jcrysgro.2010.03.028

12. M. A. R. Sarker, S. Watauchi, M. Nagao, T. Watanabe, I. Shindo, and I. Tanaka, Cryst. Growth Des. 10, 3929 (2010). http://dx.doi.org/10.1021/cg100398k

13. M. A. R. Sarker, S. Watauchi, M. Nagao, T. Watanabe, I. Shindo, and I. Tanaka, J. Cryst. Growth 317, 135 (2011). http://dx.doi.org/10.1016/j.jcrysgro.2011.01.035

14. J. H. Shin and K. H. Orr, J. Korean Assoc. Crystal Growth 5, 250 (1995).

15. W. M. Hirte and J. O. Brittain, J. Am. Ceram. Soc. 45, 546 (2006). http://dx.doi.org/10.1111/j.1151-2916.1962.tb11055.x 